University of Nebraska - Lincoln

DigitalCommons@University of Nebraska - Lincoln

CSE Conference and Workshop Papers

Computer Science and Engineering, Department

2005

\title{
DGKD: Distributed Group Key Distribution with Authentication Capability
}

Pratima Adusumilli

Xukai Zou

Byrav Ramamurthy

University of Nebraska-Lincoln, bramamurthy2@unl.edu

Follow this and additional works at: https://digitalcommons.unl.edu/cseconfwork

Part of the Computer Sciences Commons

Adusumilli, Pratima; Zou, Xukai; and Ramamurthy, Byrav, "DGKD: Distributed Group Key Distribution with Authentication Capability" (2005). CSE Conference and Workshop Papers. 59.

https://digitalcommons.unl.edu/cseconfwork/59

This Article is brought to you for free and open access by the Computer Science and Engineering, Department of at DigitalCommons@University of Nebraska - Lincoln. It has been accepted for inclusion in CSE Conference and Workshop Papers by an authorized administrator of DigitalCommons@University of Nebraska - Lincoln. 


\title{
DGKD: Distributed Group Key Distribution with Authentication Capability
}

\author{
Pratima Adusumilli, Xukaj Zou, Byrav Ramamurthy
}

\begin{abstract}
Group key management (GKM) is the most impor. tant issue in secure group communication (SGC). The existing GKM protocols fall into three typical classes: centralized group key distribution ( $C G K D$ ), decentralized group key management ( $D G K M$ ), and distributed/contributory group key agreement (CGKA). Serious problems remains in these protocols, as they require existence of central trusted entities (such as group controller or subgroup controllers), relaying of messages (by subgroup controllers), or strict member synchronization (for multiple round stepuise key agreement), thus suffering from the single point of failure and attack, performance bottheneck, or misoperations in the situation of transmission delay or network failure. In this paper, we propose a new class of GKM protocols: distributed group key distribution (DGKD). The new DGKD protocol solves the above problems and surpasses the existing GKM protocols in terms of simplicity, efficiency, scalability, and robustness.

Keywords: Secure Group Communication, Group Key Mamagement, Centralized Key Distribution, (Distributed) Contributory Key Agreement, Distributed Key Distribution.
\end{abstract}

\section{INTRODUCTION}

Secure group communications (SGC) over networks (e.g, the Internet) refers to a setting in which a group of members can send messages to and receive messages from group members, in a way that outsiders are unable to glean any information even when they are able to intercept the messages. SGC is an inseparable component of cyber security. Broad critical applications such as collaborative work, teleconferencing/medicine, multi-partner military action, and cyber forensics in critical fields depend on SGC for their security.

The most important problem facing SGC is group key management (GKM). The primary difficulty for GKM comes from member dynamics. How to design robust, scalable, efficient GKM protocols supporting high dynamics is the focus of all SGC researches. Many GKM protocols have appeared in the literature and typically fall into three categories: centralized group key distribution (CGKD), decentralized group key management with relaying (DGKM), and (distributed) contributory group key agreement (CGKA).

In CGKD schemes [1], [2], [3], [4], [5], [6], [7], [8], [9], there is a central trusted authority (called group controller

This work was partially supported by the U.S. NSF grant CCR0311577 .

P. Adusumili and X. Zou: School of Science, Purdue University at Indianapolis, Indianapolis, IN.

B. Ramamurthy: University of Nebraska-Lincoln, Lincoln, NE.
(GC)) that is responsible for generating and distributing the group key. Whenever a new member joins or an existing member leaves, the GC generates a new group key and distributes the new key to the group. The problems with the centralized schemes are the central point of failure, performance bottleneck, non-scalability, and the requirement of trustworthiness of the group controller by all members. In DGKM schemes [10], [11], [12], [13], the group is divided into multiple distinct subgroups and every subgroup has a subgroup controller (SC) responsible for key management for its subgroup. In addition, an SC has the key of its parental subgroup. When an $\mathrm{SC}$ receives a message from one subgroup, it decrypts the message, encrypts the message with the key of the other subgroup and sends to the other subgroup, i.e., relaying the message. The problems with DGKM are that SCs can still be considered as central and trusted entities (at a smaller scale) and the messages undergo multiple relaying before they reach the entire group. Relaying of every data message puts huge burden on SCs. In CGKA schemes [14], [14], [15], [16], [17], [18], the group key is generated/agreed up by uniform contributions from all group members. These kind of schemes assume equality and uniform work load among group members. They are generally executed in multiple rounds and require strict synchronization. The CGKA protocols are primarily different variations of the n-party Diffie-Hellman key agreement/exchange [14], [16], [19], [20], [17], [18]. The main problem with using this key exchange mechanism is that the group members need synchronization to iteratively form parental keys from their two children's keys. Once one member is slow or one rekeying packet is delayed, the key agreement process will be postponed or even misoperates. Moreover, there are dependances among nodes' keys (i.e., a blinded node key is dependent on the secret node key and a parental key on its two child's keys). This dependance results in the breaking of all ancestral keys once one key is compromised.

To overcome the above problems we propose a new class of GKM protocols: called distributed group key distribution (DGKD). The DGKD protocol does not assume any trusted and more powerful third party but allows the equality of capability, responsibility, and trustiness among all group members. The protocol organizes the members in a tree structure and performs any rekeying operation in just two rounds, which do not need to be strictly synchronized. 
Proceedings of the 2005 IEEE

Workshop on Information Assurance and Security

United States Military Academy, West Point, NY

The now protocol also allows strong yet siniple authentication. In addition, DGKD has the following advantages: (1) one key (not two keys) per node; (2) independance of nodes' keys; (3) robust against transmission delay, network failure or compromise of node keys. All these properties make the new protocol simple, robust, efficient and scalable.

The rest of the paper is organized as follows. Section II briefly describes the related work in the area of SGC. We propose the new protocol in Section III and the issues of performance and security are discussed in Section IV. Finally we conclude the paper in Section V.

\section{RELATED WORK}

Extensive research has been conducted on GKM and a considerable number of protocols have been developed [21], $[22],[23],[24],[25],[26],[27],[28],[29],[14],[3],[30],[31]$, $[15],[10],[32],[33],[4],[5],[16],[34],[35],[36],[37],[38]$, $[12],[7],[39],[40],[41],[17],[18],[42],[43],[44],[9],[45]$, each with different properties and performance.

SGC applications can typically be divided into broadcast/multicast communication, i.e., one sender and multiple receivers, or one-to-many communication and group (or many-to-many) communication, i.e., every sender also being a receiver. Some GKM schemes [21], [46], [15], [10], [11], [13] are suitable for broadcast applications, some other schemes $[28],[14],[15],[16] ;[41],[17],[18]$ for many-tomany applications, and there are also some schemes [3], [12], [7], [9] suitable for both kinds of applications. Based on how the group key is fornued and distributed, the GKM protocols are classified as CGKD, DGKM, and CGKA. Based on the kind of cryptosystem used, the schemes for SGC can be divided into public-key based schemes [46], [11], [13] and secret-key based schemes. Based on the kind of security, the SGC schemes may be classified as unconditionally secure or computationally secure [47], [43]. Furthermore some schemes may resist against any number of colluding adversaries, whereas others [22], [23], [24], [25], [26], [27], [32], [43] only resist against the collusion of up to certain number of adversaries. For a comprehensive survey of state-of-art techniques and challenging problems in the area of $\mathrm{SGC}$, readers are referred to the book "secure group communications over data networks", which is published by Springer [48].

Among all the GKM protocols, the tree based GKM scheme (with various variants) [1], [2], [3], [49], [19], [6], $[7],[41],[8],[9],[50],[51]$ is the most typical approach. The scheme is simple, efficient, scalable, and casy to implement. The scheme can be used for both one-to-many multicast communication as well as many-to-many group communication. Moreover the tree based GKM scheme has versions of both CGKD and CGKA.

\section{Distributed Group Key Distribution \\ (DGKD): A NEW CLASS OF GKM PROTOCOLS}

\section{A. Principle and assumption}

There are some assumptions in existing schemes. In CGKD/DGKM, a secure channel is assumed to exist between the GC/SC and each of the potential group members/subgroup members. This secure channel is generally implemented by public key cryptosystems. In CGKA, which is typically based Diffe-Hellman key exchange which suffers from the Man-in-the-Middle attack, it is assumed that each group member is equipped with some authentication capability which is also implemented by public key cryptosystems. Similarly, DGKD assumes that every group member has a publicly known (unforgeable) public key.

The new DGKD protocol adopts a tree structure and utilizes three basic mechanisms to implement distributed key generation and distribution: 1) the leaf key of a node is the public key of the corresponding group member and all the intermediate nodes' keys are secret keys, 2) the sponsor of a joining or leaving member initiates the key generation and rekeying process and sends the new keys to co-distributors (i.e., the first round), 3 ) the co-distributors then help distribute the new keys to group members in a distributed/parallel manner (i.e., the second round).

All group members have the same capability and are equally trusted. Also, they have equal responsibility, i.e. any group member could be a potential sponsor of other members or a co-distributor (depending on the relative locations of the member and the joining/leaving members in the tree). Thus there is no dependance on a single entity and even if a sponsor node fails a new sponsor for the joining/leaving member is chosen by other members. This improves the robustness of the protocol.

\section{B. Sponsor}

A sponsor is a member and the sponsor of a subtree is defined as the member hosted on the rightmost leaf in the subtree (note: "rightmost" can be equally replaced with "leftmost"). Every node has an associated sponsor field as shown in Figure 1.

The sponsor field at a particular node is updated when it is along the joining or leaving member's path. We show the joining algorithm for updating the sponsor field in Figures 2.

When a member joins, the sponsor field along the joining members path is updated from bottom to the root. If the new members id is greater than the sponsor id of the node then update the sponsor id with the new member's id. This is continued until the root (See Figure 3 ).

When $m_{7}$ joirs, the sponsor fieid along its path is updated. The sponsor id of the node $k_{6-7}$ is lesser than the id of $m_{7}$, so it is updated to 111. Similarly the sponsor id's of nodes $k_{4-7}$ and $k_{0-7}$ are updated to 111 . Whenever the 


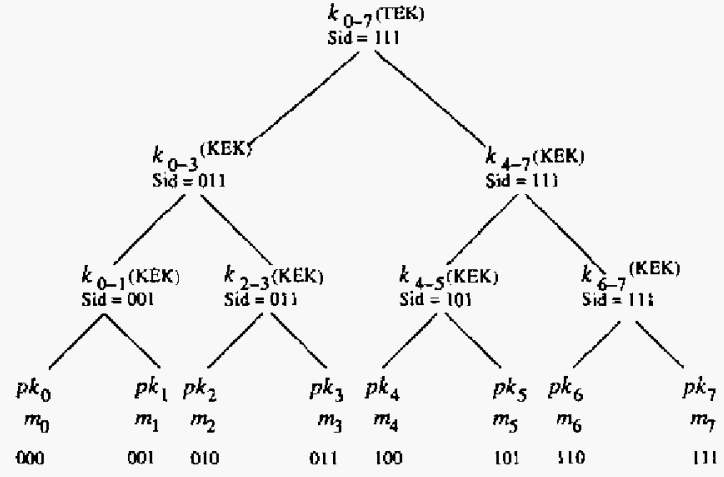

Fig. 1. A tree showing sponsor for each node

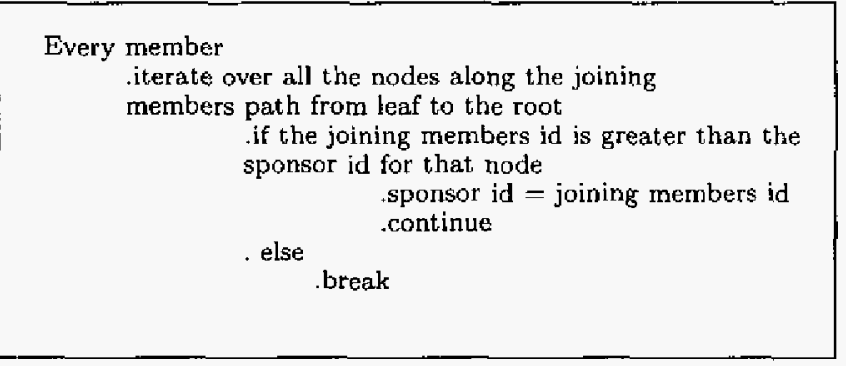

Fig. 2. Sponsor update: Join.

sponsor id for a node is greater than the joining members id then the check can be stopped.

When a member leaves, every member checks along the path of the leaving member to update the sponsor field. If a node has the leaving member as the sponsor then they update the sponsor field with the sponsor id/member id of the other child if exists. This continues upto the root (See Figure 4).

When $m_{7}$ leaves, the sponsor field along its path is updated. Since the leaving member is the sponsor all along its path, the sponsor field has to be updated by checking for the new sponsor for all the nodes. $m_{6}$ becomes the new sponsor for node $k_{6-7}$. For node $k_{4-7}$ the member ids of both its children are compared and the greater becomes the new sponsor, in this case $m_{6}$. This continues until the root.

\section{Co-distributors}

When a sponsor changes the keys along the path, it needs to distribute them. The sponsor has to distribute the keys to all the members whose keys have been changed. But it does not know the keys along the other paths to distribute the new keys. So, a co-distributor is required to distribute them. The co-distributor is the sponsor of a node on another path whose key is not known to the original sponsor. The sponsor encrypts the changed key with the co-distributors public key and broadcasts this information.

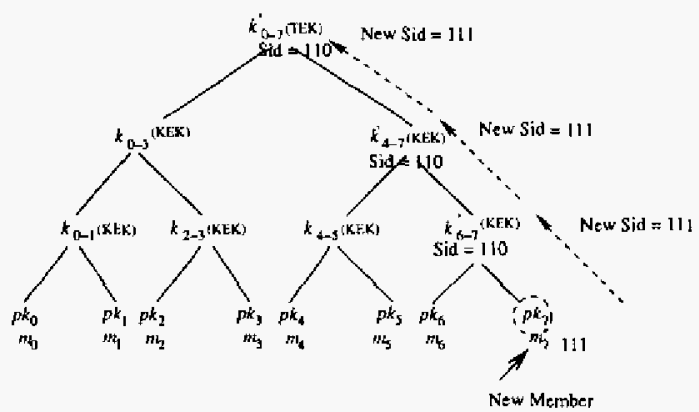

Fig. 3. Updating the sponsor field when a member joins.

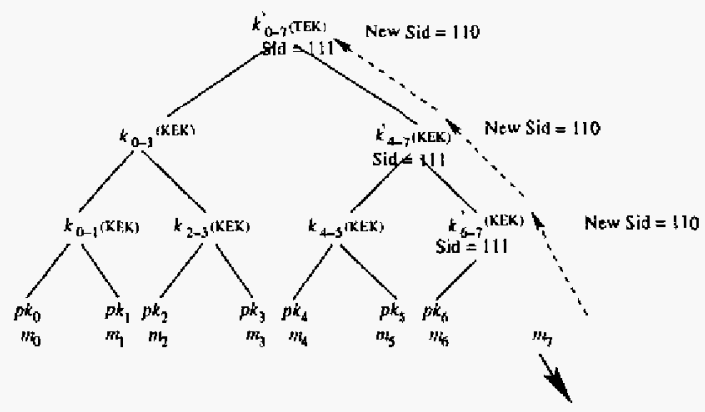

Fig. 4. Updating a sponsor field when a member leaves.

Thus, the co-distributor helps the sponsor in distributing the changed common keys along the other paths.

\section{Intial group key generation and distribution Protocol}

Suppose $n$ members $m_{1}, \ldots \ldots, m_{n}$ decide to form a group. They build a virtual key tree and selects a sponsor to decide an order in which they join the tree. Every nember updates the key tree by adding members in the key tree based on that order and they update the sponsor field in all the intermediate nodes. Then every member checks if it is responsible for generating any keys along its path. If so, it generates them and distributes the keys either directly or with the help of co-distributors. When two sponsors are responsible for generating the same key then the rightmost among them generates it. As more members join the key tree the sponsors and the height of the key tree increase.

As illustrated in Figure 5, $m_{7}, m_{5}, m_{3}$ and $m_{1}$ are responsible for generating the keys. $m n_{7}$ generates all the keys $\left(k_{6-7}, k_{4-7}\right.$ and $k_{0-7}$ ) along its path to the root. Then it encrypts as follows and broadcasts: $\left\{k_{6-7}, k_{4-7}, k_{0-7}\right\}_{p k_{6}}$, and $\left\{k_{0-7}, k_{4-7}\right\}_{p k_{5}}, m_{5}$ will decrypt $k_{0-7}$ and $k_{4-7}$ and encrypt it as $\left\{k_{0-7}, k_{4-7}\right\}_{k_{4-5}}$ where $k_{4-5}$ is generated by $m_{5}$ and sent to $m_{4}$. Similarly keys are generated by $m_{3}$ in the left subtree along its path and the root key which is generated by the rightmost sponsor $m_{7}$ is sent to the co-distributor of the left subtree $m_{3}$ as follows. $\left\{k_{0-7}\right\}_{p k_{3}}$ 


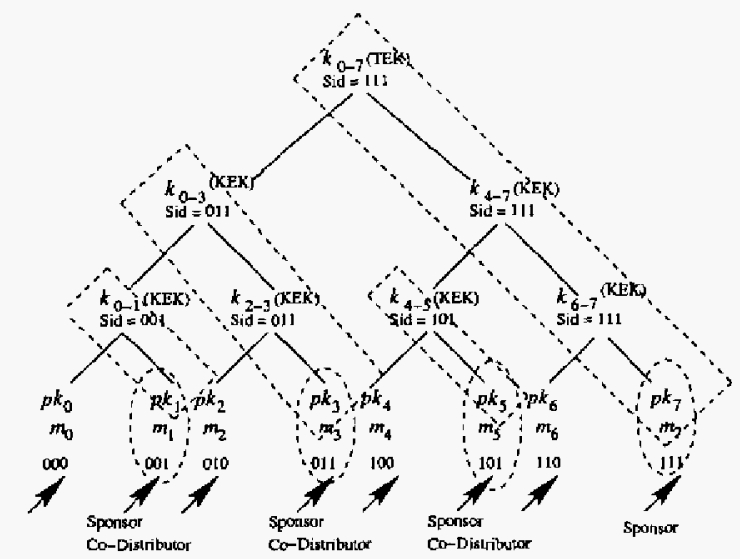

Fig. 5. Initial key generation Example

is broadcast and $m_{3}$ will decrypt $k_{0-7}$ and encrypts it as $\left\{k_{0-7}\right\}_{k_{0-3}}$ and broadcasts it. Thus every member has the newly generated keys along its path. Only two rounds are required for this protocol, one round for generating keys and distributing along the path and another for codistributors to distribute them.

\section{E. Join protocol}

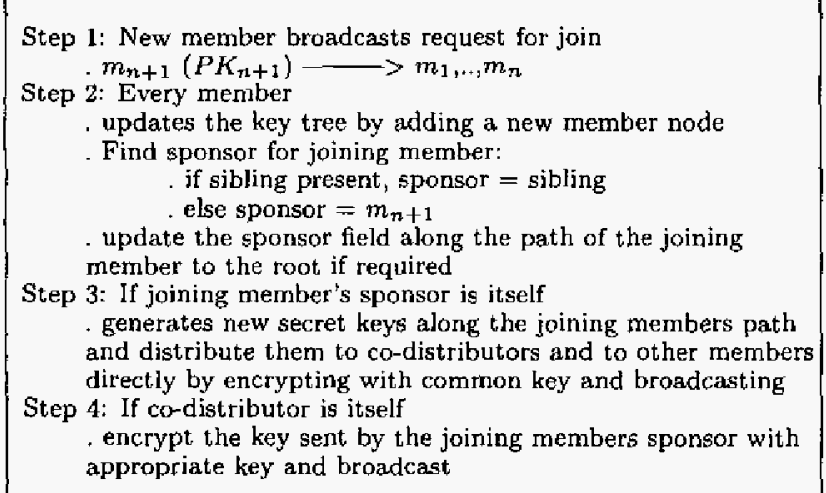

Fig. 6. Join Protocol.

Suppose there are $n$ members in the group $m_{1}, \ldots \ldots, m_{n}$. A new member $m_{n+1}$ makes a join request by broadcasting its public key PK. The rightmost member in the key tree authenticates the new member, decides the insertion location for the new member and broadcasts this information to other members. Additionally the rightmost member also sends the virtual key tree and list of public keys of other members to the new member. All other members update the key tree by adding a new member node in the specified location. Then every member checks to see if it is the sponsor of the joining member. If the new member has a sibling it becomes the sponsor and generates new keys along the path. If there is no sibling then the joining member itself
Proceedings of the 2005 IEEE

Workshop on Information Assurance and Security United States Military Academy, West Point, NY

becomes the sponsor and generates the new keys along its path and distributes them. Members update the sponsor field appropriately if required. Figure 6 describes the join protocol and Figure 7 shows the protocol operation when a new member joins.

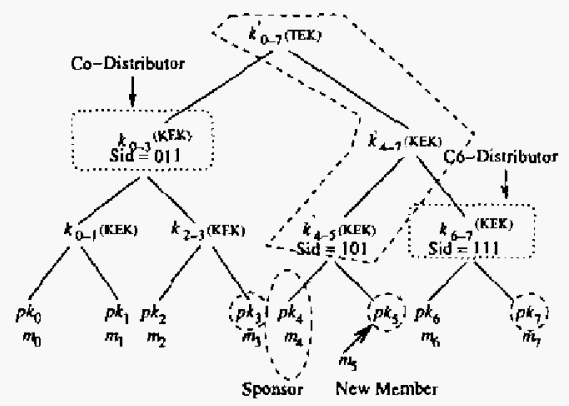

Fig. 7. A new member joins (becomes $m_{5}$ ), $m_{4}$ is sponsor and $m_{3}$ and $m_{7}$ are co-distributors.

When a new member joins, $m_{7}$ determines the position(i.e., $m_{5}$ ) and places the member there. $m_{7}$ broadcasts the position of the new member to other members. All members also determine that $m_{4}$ is the sponsor of $m_{5}$. So $m_{4}$ initiates the rekeying process as follows: 1) generates new keys $k_{4-5}^{\prime}, k_{4-7}^{\prime}$, and $\left.k_{0-7}^{\prime}, 2\right)$ after determining the co-distributors $m_{3}$ and $m_{7}$, encrypts as follows and broadcasts: $\left\{k_{4-7}^{\prime}, k_{0-7}^{\prime}\right\}_{p k_{7}}$, and $\left.\left\{k_{0-7}^{\prime}\right\}_{p k_{3}}, 3\right) . m_{3}$ will decrypt $k_{0-7}^{\prime}$ and encrypt it as $\left\{k_{0-7}^{\prime}\right\}_{k_{0-3}}$ and $m_{7}$ will decrypt $k_{4-7}^{\prime}$ and $k_{0-7}^{\prime}$ and encrypt them as $\left\{k_{4-7}^{\prime}\right\}_{k_{6-7}}$ and $\left\{k_{0-7}^{\prime}\right\}_{\left.k_{4-7}, 4\right) .} m_{4}$ also encrypts and sends the keys to $m_{5}$ as $\left\{k_{4-5}^{\prime}, k_{4-7}^{\prime}, k_{0-7}^{\prime}\right\}_{p k_{5}}$. As a result, all the members will get the new keys.

When a new member joins, only the keys along its path to the root have to be changed and distributed, which can be achieved in two rounds with atmost $\log _{2} n$ keys being changed.

\section{F. Leave protocol}

Step 1: Every member updates the key tree by removing the leaving member node . updates the sponsor field appropriately along the leaving members path if required

- determines the sponsor for changing keys along the leaving members path

Step 2: If sponsor of the leaving member is itself - generates new secret keys along the path and distributes them to co-distributors and directly to other members

Step 3: If co-distributor is itself

. broadcasts the key sent by the leaving members sponsor by encrypting it with the appropriate key

Fig. 8. Leave Protocol 
Assume that member $m_{i}$ leaves the group. Every member updates the key tree by deleting node $m_{i}$ and updates the sponsor field along the path if required. Then they determine the sponsor who generates new keys along the leaving members path and distributes them. If the leaving member does not have a sibling then the first sponsor along the leaving members path becomes responsible for changing the keys along the leaving member's path (See Figure 8).

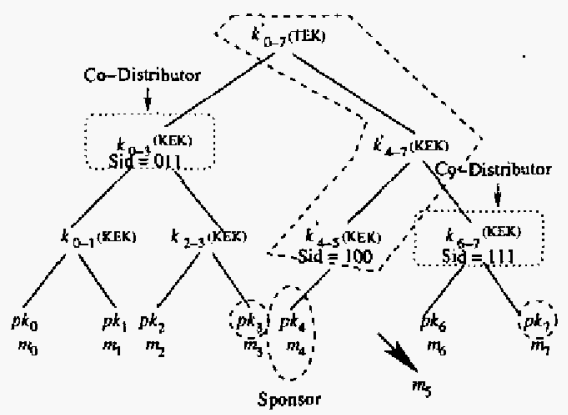

Fig, 9. A member $m_{5}$ leaves.

As shown in Figure 9 , when a member $m_{5}$ leaves, all the members will remove the node and determine that $m_{4}$ is the sponsor of $m_{5}$. So $m_{4}$ initiates the rekeying process as follows: 1) generates new keys $k_{4-5}^{\prime}, k_{4-7}^{\prime}$, and $k_{0-7}^{\prime} .2$ ) after determining the co-distributors $m_{3}$ and $m_{7}$, encrypts as follows and broadcasts: $\left\{k_{4-7}^{\prime}, k_{0-7}^{\prime}\right\}_{p k_{7}}$, and $\left.\left\{k_{0-7}^{\prime}\right\}_{p k_{3}}, 3\right)$. $m_{3}$ will decrypt $k_{0-7}^{\prime}$ and encrypt it as $\left\{k_{0-7}^{\prime}\right\}_{k_{0-3}}$ and $m_{7}$ will decrypt $k_{4-7}^{\prime}$ and $k_{0-7}^{\prime}$ and encrypt them as $\left\{k_{4-7}^{\prime}\right\}_{k_{6-7}}$ and $\left.\left\{k_{0-7}^{\prime}\right\}_{k_{4-7}}, 4\right)$. As a result, all the members will get the new keys.

When a member leaves only the keys along its path to the root have to be changed and distributed, which can be achieved in two rounds with at most $\log _{2} n$ keys being changed.

\section{G. Multiple join protocol}

Suppose $m$ new nembers join, they make a join request by broadcasting their public keys. The rightmost member in the key tree authenticates the new members, decides the locations for all the new members such that minimal number of keys are changed and broadcasts this information to other existing group members. The rightmost member also sends the virtual key tree and existing members public keys to the joining members. Every member upon receiving this message updates its key tree by adding $m$ new nodes in the determined positions. In order to perform multiple joins in one aggregate operation, it is required to find the common keys shared by the joining members in an efficient way. To achieve that we use an already proposed scheme, an efficient and scalable key tree based dynamic
Step 1: Every member

updates key tree by adding new member nodes - updates the sponsor field along all the paths of the joining members

computes the keys that need to be changed

determines the sponsors who are responsible for changing these keys

Step 2: If sponsor for one of the joining members is itself . changes the secret keys along the joining members path and distributes them to co-distributors and directly to other members

if same key has to be changed, check if right sponsor is itselt if rightmost sponsor, change the key and distribute

Step 3: If co-distributor is itself

. broadcasts the key sent by the joining members sponsor by encrypting it with the appropriate key

Fig. 10. Multiple Join Protocol.

conferencing scheme called KTDC in [52] which uses an efficient algorithm for computing the shared keys. There will be multiple sponsors responsible for changing the necessary keys. But here the shared keys which both sponsors have in common and which need to be changed will be changed by the rightmost sponsor among the sponsors (See Figure 10).

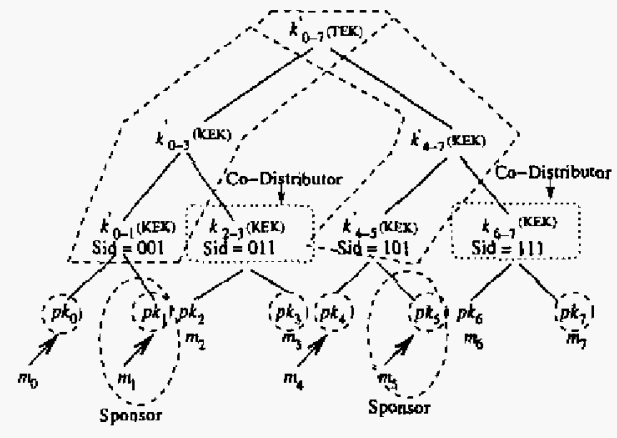

Fig. 11. New members $m_{0}, m_{1}, m_{4}$ and $m_{5}$ join.

As shown in Figure 11, when new members join, $m_{7}$ will determine the available positions (i.e., $m_{0}, m_{1}, m_{4}, m_{5}$ ) and place the members there. $m_{7}$ broadcasts this information to other group members. All members also know that $m_{5}$ is the sponsor of $m_{4}$ and $m_{1}$ is the sponsor of $m_{0}$. They also know that $m_{3}$ and $m_{7}$ are responsible for sending the key tree structure and the public key list to the joining members. $m_{5}$ initiates the rekeying process as follows: 1) generates new keys $k_{4-5}^{\prime}, k_{4-7}^{\prime}$, and $k_{0-7}^{\prime} .2$ ) after determining the co-distributors $m_{3}$ and $m_{7}$, encrypts as follows and broadcasts: $\left\{k_{4-7}^{\prime}, k_{0-7}^{\prime}\right\}_{p k_{7}}$, and $\left\{k_{0-7}^{\prime}\right\}_{p k_{3}}, 3$ ). $m_{3}$ will decrypt $k_{0-7}^{\prime}$ and encrypt it as $\left\{k_{0-7}^{\prime}\right\}_{k_{0-3}^{\prime}}$ and $m_{7}$ will decrypt $k_{4-7}^{\prime}$ and $k_{0-7}^{\prime}$ and encrypt them as $\left\{k_{4-7}^{\prime}\right\}_{k_{6-7}}$ and $\left.\left\{k_{0-7}^{\prime}\right\}_{k_{4-7}}, 4\right) . m_{5}$ also encrypts and sends the keys to $m_{4}$ as $\left\{k_{4-5}^{\prime}, k_{4-7}^{\prime}, k_{0-7}^{\prime}\right\}_{p k_{4}}$. Similarly $m_{1}$ regenerates the 
keys along its path except for the root key which should be changed by the rightmost sponsor $m_{5}$. Both $m_{1}$ and $m_{5}$ do these operations in parallel. As a result, all the members whose keys have been changed will get the new keys.

Since all the operations are done in parallel, rekeying can be achieved in two rounds by all the sponsors

When a network event causes all the previously occurred partitions to reconnect this is called a mergo. Merge is similar to multiple join and this can also be achieved in two rounds which is better than that in TGDH.

\section{H. Multiple leave protocol}

Step 1: Every member

- updates the key tree by removing all leaving member nodes updates the sponsor field along all the leaving members paths if required

determines the sponsors responsible for changing the keys along the patbs

Step 2: If sponsor for one of the leaving member is itself - generates new secret keys and distributes them to co-distributors and other members directly

if same key has to be changed, check if right sponsor is itsel if right tnost sponsor, change the key and distribute

Step 3: If co-distributor is itself

broadcasts the key sent by the leaving members sponsor by encrypting it with the common key

Fig. 12. Multiple Leave Protocol.

When multiple members leave, every member updates its key tree by deleting those momber nodes and the sponsor fields along all the paths. Then they determine the keys that need to be changed and the sponsors responsible for changing those keys. There will be multiple sponsors and each sponsor regenerates the keys and distributes them. If two sponsors are responsible for changing the same key then the rightmost among the sponsors will change the key (See Figure 12).

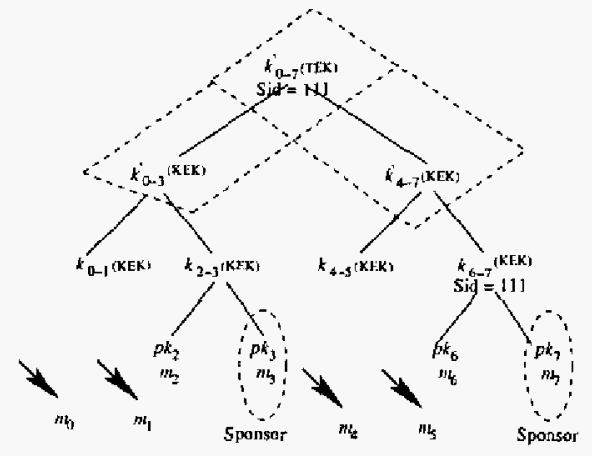

Fig. 13. Members $m_{0}, m_{1}, m_{4}$ and $m_{5}$ leave

As shown in Figure 13, when several nnembers $m_{0}, m_{1}$, $m_{4}$ and $m_{5}$ leave, every member updates its key tree by deleting those member nodes. Every member also determines that $m_{3}$ and $m_{7}$ are the sponsors. $m_{7}$ initiates the rekeying process as follows: 1) generates new keys $k_{4-7}^{\prime}$, and $\left.k_{0-7}^{\prime} .2\right)$ encrypts the new keys as follows and broadcasts: $\left\{k_{4-7}^{\prime}, k_{0-7}^{\prime}\right\}_{k_{6-7}}$ and $\left.\left\{k_{0-7}^{\prime}\right\}_{p k_{3}}, 3\right) m_{3}$ will decrypt $k_{0-7}^{\prime}$ and encrypt it as $\left\{k_{0-7}^{\prime}\right\}_{k_{0-3}^{\prime}}$ and broadcasts it. Similarly $m_{3}$ generates the keys $k_{0-3}^{\prime}$ and encrypts it with $k_{2-3}$ and broadcasts it. Both $m_{3}$ and $m_{7}$ do these operations in parallel. As a result, all the members whose keys have been changed will get the new keys.

In case of a network failure which causes disconnectivity, the group gets split and this partition can be dealt with as a multiple leave operation. Thus, even for network partition the protocol requires only two rounds for regenerating and distributing the keys. This is a great improvement compared to TGDH which requires several rounds.

\section{Authentication in $D G K D$}

Most CGKA protocols do not contain an authentication component. Furthermore, the authenticated CGKA protocols [53], [54], [55], [56], [57] are non-scalable and/or non-dynarnic. In contrast, the new DGKD protocol is not. only scalable and dynamic but also able to provide easy and strong authentication. Consider two scenarios: (1) the sponsor $m_{4}$ transmits a new key $k_{0-7}^{\prime}$ to a co-distributor $m_{3}$. (2) $m_{3}$ transmits the key $k_{0-7}^{\prime}$ to members $m_{0}, m_{1}, m_{2}$ who are in the responsibility scope of $m_{3}$. In the first case, $m_{4}$ signs the key $k_{0-7}^{\prime}$ (using $m_{4}$ 's private key), encrypts both $k_{0-7}^{t}$ and the signed $k_{0-7}^{f}$ (using $m_{3}$ 's public key $p k_{3}$ ), and sends the result to $m_{3} . m_{3}$ after receiving the message, decrypts $k_{0-7}^{\prime}$ and then verifies $m_{4}$ 's signature. In the second case, $m_{3}$ signs $k_{0-7}^{\prime}$ (by its private key), encrypts both $k_{0-7}^{\prime}$ and the signed $k_{0-7}^{\prime}$ (using $k_{0-3}$ which covers $m_{0}$ to $\left.m_{3}\right)$. Then each of the members from $m_{0}$ to $m_{2}$ can verify $m_{3}$ 's signature.

\section{Discussions}

We discuss the performance and security of our protocol in this section and analyze the communication and computation costs for join, leave, multiple join and multjple leave operations. Tree based Group Diffie-Hellmar (TGDH) [19], [34] is one of the most typical CGKA protocols in terms of efficiency and scalability, so we focus on the comparison between DGKD and TGDH.

Key generation is independent, i.e., only the sponsor is involved, thus there is no need for synchronization with other members which is required in TGDH. In this sense, DGKD is more resilient to network congestion, delay and failure than TGDH. DGKD also has strong yet simple authentication. It is also collusion free because the new keys are independent of the old keys and no matter how many members collude they cannot get the keys. Thus, it is unconditionally secure. Both TGDH and DGKD require 
Proceedings of the 2005 IEEE Workshop on Information Assurance and Security United States Military Academy, West Point, NY

two rounds for single join and leave operations. As for multiple join and leaving operations, DGKD requires two rounds but TGDH requires $\log (p)$ rounds where $p$ is the number of members involved. DGKD uses public key encryption for sending the keys to co-distributors and secret key encryption for further distribution of keys (from the codistributors to the members). TGDH requires performing modular exponentiations which is in the same complexity as the public key encryption. In summary, DGKD is comparable and in some cases better than TGDH in terms of communication and computation costs.

\section{Conclusion}

We proposed a new class of GKM protocols for SGC with strong yet simple authentication capability. The proposed protocol solves some serious problems in the existing protocols and is simple, robust, efficient, and scalable. The future work is to implement and test the new protocol.

\section{REFERENCES}

(1) R. Canetti, J. Garay, G. Itkis, D. Micciancio, M. Naor, and B. Pinkas, "Multicast security: a taxonomy and some efficient constructions," Proceedings of INFOCOM'99: Conference on Computer Communications, vol. 2, pp. 708-716, Mar. 1999.

[2] R. Canetti, T. Malkin, and K. Nissim, "Efficient communicationstorage tradeoffs for multicast encryption," Lecture Notes in Computer Science (Advances in Cryptology-EUROCRYPT'99), vol. 1592 , Pp. $459-470,1999$

3] G. Caronni, K. Waldvogel, D. Sun, and B. Plattner, "Efficient security for large and dynamic multicast groups," Proceedings of the Seventh IEEE International Workshop on Enabling Technologies: Infrastructure for Collaborative Enterprises (WETICE' '98) (Cat. No.98TB100253), pp. 376-383, June 1998.

[4] H. Hamey and C. Muckenhim, "Group Key Management Protocol (GKMP) Architecture," RCF 209/, July 1997

[5] H. Hamey and C. Muckenhim, "Group Key Management Proto col (GKMP) Specification," RCF 2093, July 1997.

[6] H. Harney and E. Harder, "Logical key hierarchy protocol," Internet Draft (work in progress), draft-harney-sparta-lkhp-sec00.txt, Intemet Engineering Task Force, Mar. 1999.

[7] G. Noubir, "Multicast security," European Space Agency, Praject: Performance Optimization of Intemet Protocal Vio Satellite, Apr. 1998

[8] D. Wallner, E. Harder, and R. Agee, "Key management for multicast: Issues and architectures," Internet Draft (work in pragress), draft-wallner-key-arch-a1.txt, Internet Eng. Task Force, Sept. 1998.

(9] C. K. Wong, M. Gouda, and S. S. Lam, "Secure group communications using key graphs," SIGCOMM 'g8, Also University of Texas at Austin, Computer Science Technical report TR 97-29, pp. 68-79, Dec. 1998.

[10] L. R. Dondeti, S. Mukherjee, and A. Samal, "A dual encryption protocol for scalable secure multicasting," In Fourth IEEE Symposium on Computers and Communications, pp. 2-8, July 1999.

[11] F. Du, L. M. Ni, and A. H. Esfahanian, "Towards solving multicast key management problem," ICCCN'99 Eighth International Conference on Computer Comimunications and Networks, pp. $232-236$, Oct. 1999

[12] S. Mittra, "Iolus: A framework for scalable secure multicasting," Journal of Computer Communication Reviews, vol. 27, no. 4, pp. 277-288, 1997.

[13] R. Molva and A. Pannetrat, "Scalable multicast security in dynamic groups," 6th ACM Conference on Computer and Commu. nications Security (ACM CCS 1999), Singapore, pp. 101-112, Nov. 1999.
[14] M. Burmester and Y. Desmedt, "Efficient and secure conferencekey distribution," Security Protocols Workshop. pp. 119-129, 1996.

[15] L. R. Dondeti, "Efficient private group communication over public networks," Phd. Dissertation, CSE UNL, 1999.

[16] I. Ingemarsson, D. Tang, and C. Wong, "A conference key distribution system," IEEE Thansactions on Information Theory, vol. 28, pp 714-720, Sept. 1982.

[17] D. Steer, L. Strawczynski, W. Diffie, and M. Wiener, "A secure audio teleconference system," Advances in CryptologyCRYPTO'88, LNCS, Springer-Verlag, vol. 403, pp. 520-528, Aug. 1990.

[18] M. Steiner, G. Tsudik, and M. Waidner, "Diffie-Hellman key distribution extended to group communication," $A C M$ Conference on Computer and Communications Security (ACM CCS 1996) pp. 31-37, Mar. 1996.

[19] Y'. Kim, A. Perrig, and G. Tsudik, "Simple and fault-tolerant key agreement for dynamic collaborative groups," In Proceedings of the 7th ACM Conference on Computer and Communications Security (ACM CCS 2000), pp. 235-244, Nov. 2000

[20] Y. Kim, A. Perrig, and G. Tsudik, "Communication-efficient group key agreement," In Information System Security, Proceedings of the 17th International Information Security Conference IFYP SECOI, pp. 229-244, June 2001.

[21] A. Bakkardie, "Scalable multicast key distribution," RFC 1949 , 1996

[22] A. Beimel and B. Chor, "Interaction in key distribution schemes," Advances in Cryptology - CRYPTO'93, LNCS, Springer, Berlin, vol. 773, pp. 444-457, 1994

[23] A. Beimel and B. Chor, "Communications in key distribution schemes," IEEE Transactions on Information Theory, vol. 42, pp. $19-28,1996$

[24) R. Blom, "An optimal class of symmetric key generation systems," Advances in Cryptology - EUROCRYPT'84, LNCS, Springer, Berlin, vol. 209, pp. 335-338, 1985.

[25] C. Blundo and A. Cresti, "Space requirements for broadcast encryption," Advances in CTyptology - EUROCRYPT'94, LNCS, Springer, Berlin, vol. 950, pp 287-298, 1995

[26] C. Blundo, L. A. F. Mattos, and D. R. Stinson, "Generalized Beimel-Chor scheme for broadcast encryption and interactive key distribution," Theoretical Computer Science, vol. 200, pp. 313-334, June 1998.

[27] C. Blundo, A. D. Santis, A. Herzberg, S. Kutten, U. Vaccaro, and M. Yung, "Perfect secure key distribution for dynamic conferences," Advances in Cryptology - CRYPTO'g2, LNCS, Springer, Berlin, vol. 740, pp. 471-486, Aug. 1993.

[28] M. Burmester and Y. Desmedt, "A secure and efficient conference key distribution system," Advances in Cryptology - EUROCRYPT'94, LNCS, Springer, Berim, vol. 950, pp. 275-286, May 1995

[29] J. F. Bob Briscoe, "Nark: receiver-based multicast nonrepudiation and key management," Proceedings of the 1 st ACM conference on Electronic commerce, pp. 22-30. Nov. 1999.

[30] Y. Challal, H. Bettahar, and A. Bouabdallah, "Sakm: a scalable and adaptive key management approach for multicast communications," ACM SIGCOMM Computer Communication Review, vol. 34 , pp. 55-70, Apr. 2004

[31] W. Chen and L. R. Dondeti, "Recommendations in using group key management algorithms," DAFPA Information Survivability Conference and Exposition, vol. 2, pp. 222-227, Apr. 2003.

[32] A. Fiat and M. Naor "Broadcast encryption" Advances in Cryptology - CRYPTO'9S, LNCS, Springer, Berlin, vol. 773, pp. $480-491,1994$.

[33] S. M. Ghanem and H. Abdel-Wahab, "A secure group key management framework: Design and rekey issues," Eighth IEEE International Symposium on Computers and Communications, pp. 797-802, June 2003

[34] Y. Kim, A. Perrig, and G. Tsudik, "Tree-based group key agreement," ACM Transactions on Information Systems Security, vol. 7, pp. 60-96, Feb. 2004

[35] F.-Y. Lee and S. Shieh, "Scalable and lightweight key distribution for secure group communications, "Intemational Jourrial of Network Management, vol. 14, pp. 167-176, May 2004. 
[36] J.-C. Lin, C.-Y. Chou, F. Lai, and K.-P. Wu, "A distributed key management protocol for dynamic groups," 27 th Annual IEEE Conference on Local Computer Networks (LCN'O2), pp. 01130122, Nov. 2002.

[37] D. Liu, P. Ning, and K. Sun, "Cryptographic protocols/ network security: Efficient self-healing group key distribution with revocation capability," Proceedings of the $10 \mathrm{th}$ ACM conference on Computer and communication security, pp. 231-240, Oct. 2003.

[38] C. Meadows and P. Syverson, "Group key management and sig. natures: Formalizing gdoi group key management requirements in npatrl," Proceedings of the 8th ACM conference on Computer and Communications Security, pp. 235-244, Nov. 2001.

[39] C.-S. Park and D.-H. Lee, "Secure and efficient key management for dynamic multicast groups," ACM SIGOPS Operating Systems Review, vol. 35, pp. 32-38, Oct. 2001.

[40] S. Rafaeli and D. Hutchison, "Hydra: A decentralised group key management," Eleventh IEEE International Workshops on Enabling Technologies: Infrastructure for Callaborative Enterprises (WETICE'O2), pp. 62-67, June 2002.

[41] A. T. Sherman and D. A. McGrew, "Key establishment in large dynamic groups using one-way function trees," IEEE transactions on Softwore Engineering, vol. 29, pp. 444-458, May 2003

[42] M. Steiner, G. Tsudik, and M. Waidner, "Key agreement in dynamic peer groups," IEEE Transactions on Parallel and Distributed Systems, vol. 11, pp. 769-780, Aug. 2000.

[43] D. R. Stinson, "On some methods for unconditionally secure key distribution and broadcast encryption," Design, Codes and Cryptography, vol. 12, pp. 215-243, June 1997.

[44] Y.M. Tseng, "A scalable key-management scheme with minimizing key storage for secure group communjcations," International Journal of Network Maragement, vol. 13, pp. 419-425, Nov. 2003.

[45] S. Zhu, S. Setia, and S. Jajodia, "Performance optimizations for group key management schemes," 23rd International Conference on Distributed Computing Systems, pp. 163-171, May 2003

[46] G. H. Chiou and W.T.Chen, "Secure broadcasting using the Secure Lock," IEEE Transactions on Software Engineering, vol. 15, pp. 929-934, Aug. 1989

[47] D. R. Stinson, ed., Cryptography: Theory and Practice. Boca Raton, Florida, USA: CRC Press, Inc., 1995.

[48] X. Zou, B. Ramamurthy, and S. S. Magliveras, eds., Secure Group Communications over Data Networks. New York, NY, USA, ISBN: 0-387-22970-1 (The ebook ISBN: 0-387-22971-X) Springer, Oct. 2004.

[49] L. R. Dondeti, S. Mukherjee, and A. Samal, "DISEC: a distributed framework for scalable secure many-to-many communication," In Proceedings of Fifth IEEE Symposium on Computers and Communications (ISCC 2000), pp. 693-698, July 2000

[50] X. B. Zhang, S. S. Lam, D.-Y. Lee, and Y. R. Yang, "Protocol design for scalable and reliable group rekeying," Proceeding $S P I E$ Conference on Scalability and Traffic Control in IP Networks, pp. 87-108, Aug. 2001

[51] X. Zou and B. Ramamurthy, "A block-free tree-based group Diffie-Hellman key agreement for secure group communications," Proceedings of International Conference on Parallel and Distributed Computing and Networks, Innsbrick, Austria, pp. 288293, Feb. 2004

[52] X. Zou, S. Magliveras, and B. Ramamurtby, "Key tree based scalable sccure dynamic conferencing schemes," Proceedings of International Conference on Parallel and Distributed Computing and Systems (PDCS 2004), MIT Cambridge, MA, USA, November 9-11, pp. 61-66, Nov. 2004

[53] G. Ateniese, M. Steiner, and G. Tsudik, "Authenticated group key agreement and friends," Proceedings of the 5 th $A C M$ conference on Computer and communications security, CA, USA, pp. 17-26, 2008 .

[54] E. Bresson, O. Chevassut, and D. Pointcheval, "Dynarnic group Diffie-Hellman key exchange under standard assumptions," $\mathrm{Ad}$ vances in Cryptology - EUROCRYPT'2002, LNCS, Springer Berlin, vol. 2332, pp. 321-336, 2002.

[55] E. Bresson, O. Chevassut, D. Pointcheval, and J.-J. Quisquater "Provably authenticated group Diffe-Hellman key exchange,"
Proc. 8th Annual ACM Conference on Computer and Communications Security (COS 01 ), pp. 255-264, 2001

[56] J. Katz and $M$. Yung, "Scalable protocols for authenticated group key exchange," The 23rd Annual International Cryptology Conference, CRYPTO 2003, CA, USA, Aug. 2003.

[57] H.-J. Kim, S.-M. Lee, and D. H. Lee, "Constant-round authenticated group key exchange for dynamic groups," A siacrypto'04, http://www iris.re.kr/ac04/data/Asiacrypt2004 106\%20Key\%20Management/02_Hyuri-Jeong\%20Kim pdf 2004 . 